\title{
Detection and Parameter Estimation of Multicomponent LFM Signal Based on the Cubic Phase Function
}

\author{
Yong Wang and Yi-Cheng Jiang \\ Harbin Institute of Technology, Research Institute of Electronic Engineering Technology, Harbin 150001, China \\ Correspondence should be addressed to Yong Wang, wangyong6012@hit.edu.cn
}

Received 27 September 2007; Revised 17 January 2008; Accepted 5 March 2008

Recommended by Jar-Ferr Yang

\begin{abstract}
A new algorithm for the detection and parameters estimation of LFM signal is presented in this paper. By the computation of the cubic phase function (CPF) of the signal, it is shown that the CPF is concentrated along the frequency rate law of the signal, and the peak of the CPF yields the estimate of the frequency rate. The initial frequency and amplitude can be obtained by the dechirp technique and fast Fourier transform. And for multicomponent signal, the CLEAN technique combined with the CPF is proposed to detect the weak components submerged by the stronger components. The statistical performance is analyzed and the simulation results are shown simultaneously.
\end{abstract}

Copyright (c) 2008 Y. Wang and Y.-C. Jiang. This is an open access article distributed under the Creative Commons Attribution License, which permits unrestricted use, distribution, and reproduction in any medium, provided the original work is properly cited.

\section{INTRODUCTION}

Linear frequency-modulated (LFM), or chirp, signals are frequently encountered in applications such as radar, sonar, bioengineering, and so forth. The amplitude, initial frequency, and chirp rate are the basic parameters which denote the characteristic of the LFM signal, and the estimation of them is an important problem in the signal processing community. Several estimation procedures have been proposed, but most are based on the maximum likelihood (ML) principle $[1,2]$. These methods can be ascribed to a multivariable optimization algorithm and the accuracy of them strongly depends on the grid resolution in the search procedure. The computational burden may be too high to obtain reasonable accuracy. In recent years, many techniques based on the time-frequency analysis have been presented to solve this problem, such as the Wigner-Hough transform $[3,4]$, the Radon-ambiguity transform [5], and the fractional Fourier transform (FrFt) [6], and so forth. These techniques alleviate the computational burden in a way, but still need complex searching and the application of them is limited. Hence, the fast estimation of the parameter of LFM signal with high accuracy is still an urgent problem to us all. In this paper, a new algorithm of detection and parameters estimation of LFM signal is presented, by the computation of the CPF [7] of the signal, it is shown that the CPF is concentrated along the frequency rate law of the signal, and the estimation of the frequency rate can be obtained by finding the CPF peak. Then the estimation of the initial frequency and amplitude can be implemented by the dechirp technique and fast Fourier transform. The algorithm requires only one-dimensional (1D) maximizations, which lessen the computational burden greatly. And for multicomponent signal, the CLEAN technique combined with the CPF is proposed to detect the weak components submerged by the stronger components, and the combination technique is valuable in practice. The statistical performance is analyzed at last and the simulation results demonstrate the validity of the algorithm proposed.

\section{DETECTION AND PARAMETER ESTIMATION OF LFM SIGNAL BASED ON CPF}

The cubic phase function $(\mathrm{CPF})$ was introduced in [8] for the purpose of estimating the instantaneous frequency rate law of a quadratic FM signal. In this paper, a new algorithm for detection and parameter estimation of multicomponent LFM signal based on the CPF is developed in the following.

For a monocomponent LFM signal

$$
s(t)=b e^{j \phi(t)}=b e^{j\left(\alpha t+\beta t^{2}\right)},
$$




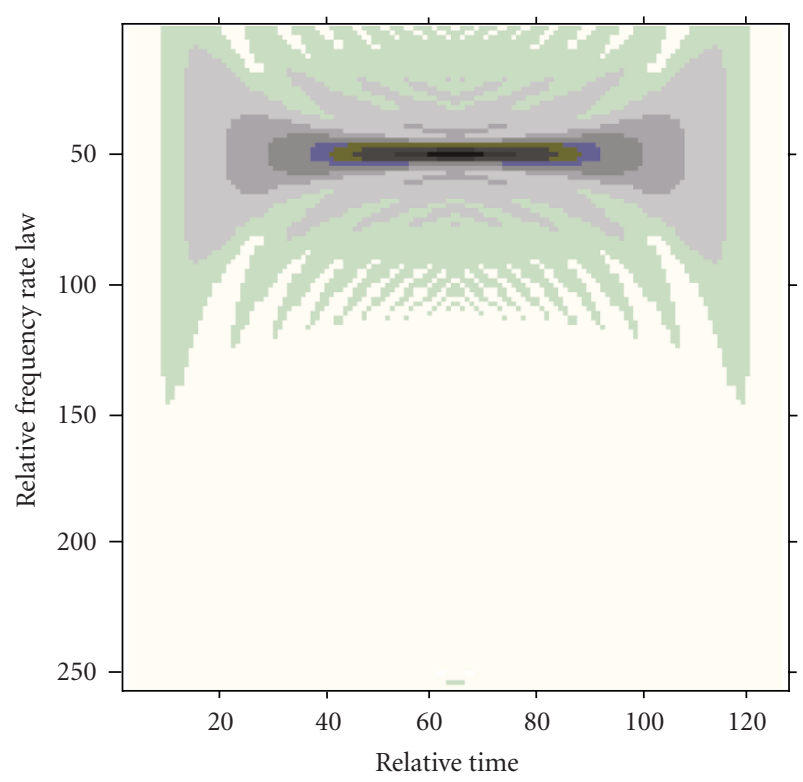

(a) 2D distribution

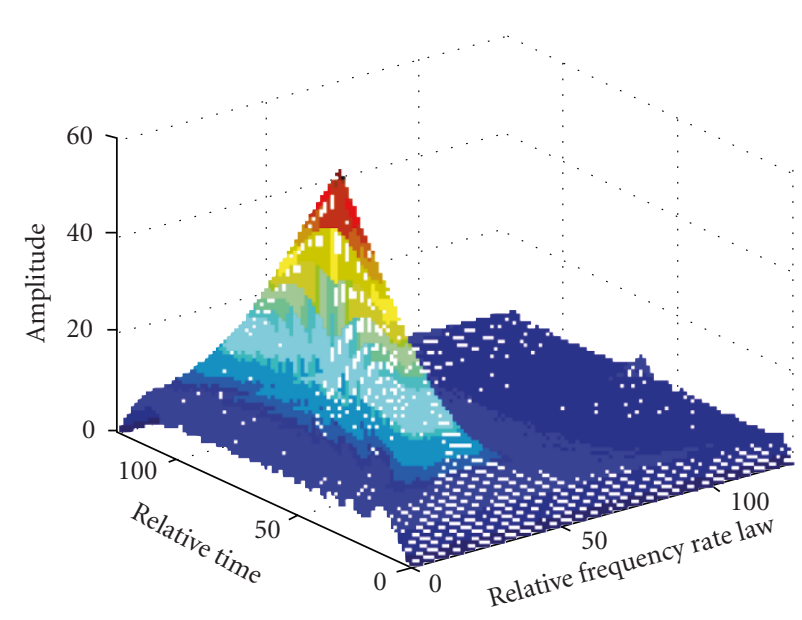

(b) 3D distribution

FIgure 1: The CPT of a LFM signal.

where $\phi(t)$ is the signal phase, $b$ is amplitude, $\alpha$ is initial frequency, and $\beta$ is chirp rate. The CPF is defined as

$$
\mathrm{CP}(t, u)=\int_{0}^{+\infty} s(t+\tau) s(t-\tau) e^{-j u \tau^{2}} d \tau
$$

By substituting (1) in (2), we obtain

$$
\mathrm{CP}(t, u)=b^{2} e^{2 j\left(\alpha t+\beta t^{2}\right)} \int_{0}^{+\infty} e^{j(2 \beta-u) \tau^{2}} d \tau .
$$

Using the identity

$$
\int_{-\infty}^{+\infty} e^{-j m t^{2}} d t=\sqrt{\frac{\pi}{m}} e^{-j(\pi / 4)}, \quad m>0,
$$

we obtain

$$
|\mathrm{CP}(t, u)|= \begin{cases}\infty & u=2 \beta \\ \frac{b^{2}}{2} \sqrt{\frac{\pi}{|u-2 \beta|}} & u \neq 2 \beta .\end{cases}
$$

It is not hard to see that $\mathrm{CP}(t, u)$ peaks along the curve $u=2 \beta$, so the chirp rate $\beta$ can be estimated. Then the parameters $\alpha$ and $b$ can be estimated by dechirping and finding the Fourier transform peak. For a discrete signal in the additive noise

$$
x(n)=s(n)+v(n), \quad|n| \leq \frac{N-1}{2},
$$

where $v(n)$ is complex white Gaussian noise of zero mean and power of $\sigma^{2}$. The discrete CPF is defined as

$$
\mathrm{CP}(n, u)=\sum_{m=0}^{(N-1) / 2} x(n+m) x(n-m) e^{-j u m^{2}}
$$

The two-dimensional (2D) distribution and threedimensional (3D) distribution of the CPF for a LFM signal are shown in Figures 1(a) and 1(b), respectively. We can see from Figure 1 (a) that the CPF is concentrated along the curve $u=2 \beta$, and we can obtain the estimation of chirp rate $\beta$ at arbitrary time, but from Figure 1(b), we can see that when $n=0$, the CPF gets its maximum value, hence, the estimation of $\hat{\beta}$ can be obtained by finding the peak of $\mathrm{CP}(0, u)$. Then the estimation of $\alpha$ and $b$ can be obtained by the following two expressions:

$$
\begin{gathered}
\hat{\alpha}=\arg \max _{\alpha}\left|\sum_{n=-(N-1) / 2} s(n) e^{-j\left(\alpha n+\hat{\beta} n^{2}\right)}\right|, \\
\hat{b}=\left|\frac{1}{N} \sum_{n=-(N-1) / 2}^{(N-1) / 2} s(n) e^{-j\left(\hat{\alpha} n+\hat{\beta} n^{2}\right)}\right| .
\end{gathered}
$$

The CPF, like the ambiguity function, is bilinear. It, therefore, produces "cross-terms" when multiple components are present. So, the influence of cross-terms should be studied.

Theorem 1. For multicomponent LFM signal, there exist the "cross-terms" in the CPF, but this will not influence the detection and parameter estimation of the "autoterms."

Proof. For simplicity, we discuss here the two components case, which are modeled as

$$
s(t)=s_{1}(t)+s_{2}(t)=b_{1} e^{j\left(\alpha_{1} t+\beta_{1} t^{2}\right)}+b_{2} e^{j\left(\alpha_{2} t+\beta_{2} t^{2}\right)} .
$$


Hence, we obtain

$$
\begin{aligned}
s(t+ & \tau) s(t-\tau) \\
= & b_{1}^{2} e^{2 j\left(\alpha_{1} t+\beta_{1} t^{2}\right)} e^{2 j \beta_{1} \tau^{2}}+b_{2}^{2} e^{2 j\left(\alpha_{2} t+\beta_{2} t^{2}\right)} e^{2 j \beta_{2} \tau^{2}} \\
& +b_{1} b_{2} e^{j\left(\alpha_{1}+\alpha_{2}\right) t+j\left(\beta_{1}+\beta_{2}\right) t^{2}} e^{j\left(\beta_{1}+\beta_{2}\right) \tau^{2}+j\left(\alpha_{1}-\alpha_{2}\right) \tau+2 j\left(\beta_{1}-\beta_{2}\right) t \tau} \\
& +b_{1} b_{2} e^{j\left(\alpha_{1}+\alpha_{2}\right) t+j\left(\beta_{1}+\beta_{2}\right) t^{2}} e^{j\left(\beta_{1}+\beta_{2}\right) \tau^{2}-j\left(\alpha_{1}-\alpha_{2}\right) \tau-2 j\left(\beta_{1}-\beta_{2}\right) t \tau .}
\end{aligned}
$$

The CPF of the "autoterms" has the form of (5), peaks along the curve $u=2 \beta_{1}$ and $u=2 \beta_{2}$, respectively. Now let us compute the CPF of the "cross-terms"

$$
\begin{aligned}
& \mathrm{CP}_{\text {cro }}(t, u) \\
& =2 b_{1} b_{2} e^{j\left(\alpha_{1}+\alpha_{2}\right) t+j\left(\beta_{1}+\beta_{2}\right) t^{2}} \\
& \quad \times \int_{0}^{+\infty} e^{j\left(\beta_{1}+\beta_{2}-u\right) \tau^{2}} \cos \left[\left(\alpha_{1}-\alpha_{2}\right) \tau+2\left(\beta_{1}-\beta_{2}\right) t \tau\right] d \tau .
\end{aligned}
$$

$$
\begin{aligned}
& \text { If } u=\beta_{1}+\beta_{2} \text {, we obtain } \\
& \begin{aligned}
\left|\mathrm{CP}_{\mathrm{cro}}(t, u)\right| & =2 b_{1} b_{2}\left|\int_{0}^{+\infty} \cos \left[\left(\alpha_{1}-\alpha_{2}\right) \tau+2\left(\beta_{1}-\beta_{2}\right) t \tau\right] d \tau\right| \\
& \leq\left|\frac{2 b_{1} b_{2}}{\left(\alpha_{1}-\alpha_{2}\right)+2\left(\beta_{1}-\beta_{2}\right) t}\right| \\
& <\infty
\end{aligned}
\end{aligned}
$$

If $u \neq \beta_{1}+\beta_{2}$, we obtain

$$
\left|\mathrm{CP}_{\mathrm{cro}}(t, u)\right|=b_{1} b_{2} \sqrt{\frac{\pi}{\left|u-\left(\beta_{1}+\beta_{2}\right)\right|}} .
$$

We can see from (13) and (14) that, the CPF of the "cross-terms" is bounded, while the CPF of the "autoterms" is infinite when $u=2 \beta_{1}$ or $u=2 \beta_{2}$. So, the existence of the "cross-terms" does not influence the detection of the "autoterms."

Remark 1. The phase information of the LFM signal is neglected in this paper, because in most situations, the characteristics of LFM signal are determined by the chirp rate and initial frequency.

Remark 2. For an LFM signal with finite length, the maximum value of its CPF is finite, and the result of Theorem 1 is ideal. For a signal in practice, the conclusion above is still valid.

Remark 3. The CPF algorithm is suitable for the LFM signal with the constant amplitude, initial frequency, and chirp rate, which can be illustrated by the definition of the CPF.

Remark 4. The estimate of the chirp rate $\hat{\beta}$ can be obtained by finding the peak of $\operatorname{CP}(n, u)$ in (7) when $n=0$, which would require $O(N)$ operations. Whereas, the ML algorithm, the Wigner-Hough transform and the fractional Fourier transform (FrFt) would require $O\left(N^{2}\right)$ operations when estimating the parameters of an LFM signal.

For multicomponent signal, the CLEAN technique [9] combined with the CPF is proposed to detect the weak components submerged by the stronger components, just as follows:

Step 1. Let $k=1$, where $k$ is the number of signal components, $s(t)$ is the original signal.

Step 2. Compute the CPF of $s(t)$ according to (2), and get its absolute value $|\mathrm{CP}(t, u)|$.

Step 3. Finding the peak of $|\mathrm{CP}(t, u)|$, it is concentrated along the curve $u=2 \beta_{k}$, then the estimated values $\left\{b_{k}, \alpha_{k}, \beta_{k}\right\}$ of the $k$ th LFM component are obtained according to (8) and (9).

Step 4. Construct the reference signal $s_{r 1}(t)=\exp \left(-j \beta_{k} t^{2}\right)$, then multiply it with $s(t)$, we obtain $s_{1}(t)=s(t) s_{r 1}(t)$. Now the $k$ th LFM component has been dechirped to a sinusoidal signal, while the other components are still LFM signals.

Step 5. Design a filter with narrow bandwidth around $\alpha_{k}$, then the $k$ th component of $s_{1}(t)$ is filtered out and this operation has little influence on other components.

Step 6. Multiply the residual signal with $s_{r 2}(t)=\exp \left(j \beta_{k} t^{2}\right)$, and the other components can be calibrated to the original form. So, the residual signal without the $k$ th LFM component can be obtained.

Step 7. Let $k=k+1$, repeat Steps 2-6 until the energy of the residual signal is less than a threshold.

Figure 2 is an example to show the validity of the method above. There are two LFM components with length 255, the CPF on the plane $(t, u)$ is shown in Figure 2(a), and the CPF of the signal that has restrained the first component is shown in Figure 2(b). We can see that, on the parameter plane, the weak components will be submerged by the stronger components, and we can improve the dependability of signal detection by the CLEAN technique.

\section{STATISTICAL PERFORMANCE}

\subsection{Signal-to-noise ratio}

In the presence of signal $s(n)$ without noise, the value $\mathrm{CP}_{s}(n, u)$ has a peak at the coordinates $(0,2 \beta)$; for $x(n)=$ $s(n)+v(n)$, the value $\mathrm{CP}_{s+v}(n, u)$ becomes a random variable and there exists a variance $\operatorname{var}\left\{\mathrm{CP}_{s+v}(0,2 \beta)\right\}$. The output SNR is defined as

$$
\mathrm{SNR}_{\text {out }}=\frac{\left|\mathrm{CP}_{s}(0,2 \beta)\right|^{2}}{\operatorname{var}\left\{C P_{s+v}(0,2 \beta)\right\}}
$$




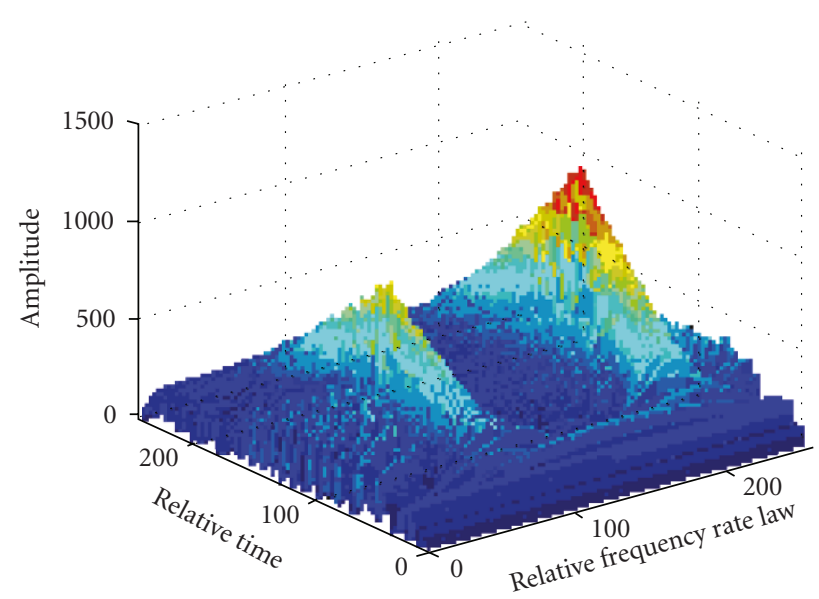

(a) The CPF on $(t, u)$ plane

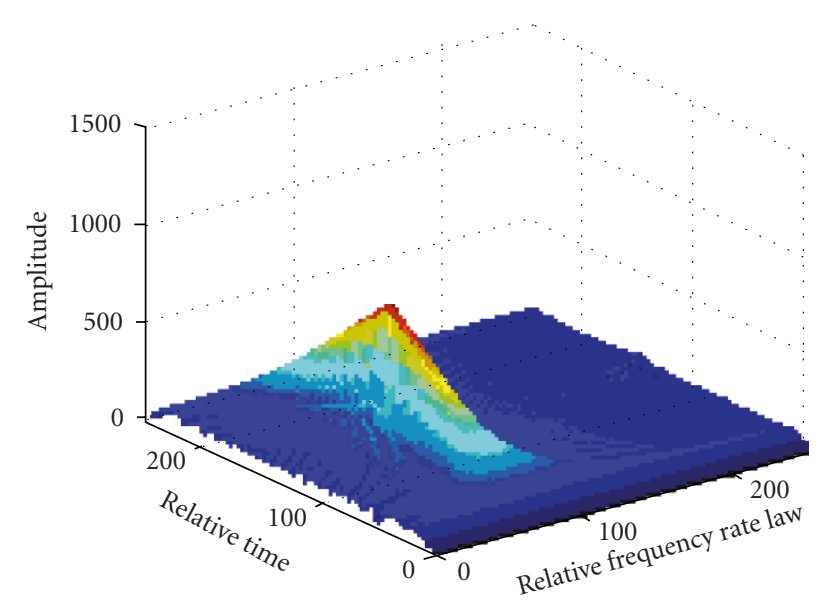

(b) The CPF of the signal that has restrained the first component

Figure 2: The signal separation based on CLEAN technique.

The expected value of $\mathrm{CP}_{s+v}(0,2 \beta)$ is

$$
\begin{aligned}
E\left\{\mathrm{CP}_{s+v}(0,2 \beta)\right\} & =\sum_{m=0}^{(N-1) / 2} E\{s(m) s(-m)\} e^{-2 j \beta m^{2}} \\
& =\frac{1}{2}(N+1) b^{2} .
\end{aligned}
$$

The second-order moment of $\mathrm{CP}_{s+v}(0,2 \beta)$ is

$$
\begin{aligned}
E\{\mid & \left.\left.\mathrm{CP}_{s+v}(0,2 \beta)\right|^{2}\right\} \\
= & \sum_{m=0}^{(N-1) / 2} \sum_{k=0}^{(N-1) / 2} E\{[s(m)+v(m)][s(-m)+v(-m) \\
& \left.\cdot\left[s^{*}(k)+v^{*}(k)\right]\left[s^{*}(-k)+v^{*}(-k)\right]\right\} e^{-2 j \beta\left(m^{2}-k^{2}\right)} \\
= & \sum_{m=0} \sum_{k=0}^{N-1) / 2(N-1) / 2}\left[s(m) s(-m) s^{*}(k) s^{*}(-k)\right. \\
& +E\left\{v(m) v^{*}(-k)\right\} s(-m) s^{*}(k) \\
& +E\left\{v(-m) v^{*}(k)\right\} s(m) s^{*}(-k) \\
& +E\left\{v(-m) v^{*}(-k)\right\} s(m) s^{*}(k) \\
& +E\left\{v(m) v^{*}(k)\right\} E\left\{v(-m) v^{*}(-k)\right\} \\
& \left.+E\left\{v(m) v^{*}(-k)\right\} E\left\{v(-m) v^{*}(k)\right\}\right] e^{-2 j \beta\left(m^{2}-k^{2}\right)} .
\end{aligned}
$$

By computing each term in (17), we obtain

$$
E\left\{\left|\mathrm{CP}_{s+v}(0,2 \beta)\right|^{2}\right\}=\left(\frac{N+1}{2}\right)^{2} b^{4}+(N+3) b^{2} \sigma^{2}+\frac{N+3}{2} \sigma^{4} .
$$

By combining (16) and (18), we obtain the variance

$$
\operatorname{var}\left\{\mathrm{CP}_{s+v}(0,2 \beta)\right\}=(N+3) b^{2} \sigma^{2}+\frac{N+3}{2} \sigma^{4} .
$$

Hence, we can express the output SNR as a function of the input SNR,

$$
\mathrm{SNR}_{\text {out }} \approx \frac{(N / 2) \mathrm{SNR}_{\text {in }}^{2}}{2 \mathrm{SNR}_{\text {in }}+1}
$$

where $\mathrm{SNR}_{\text {in }}=b^{2} / \sigma^{2}$ is the input SNR.

We can see from (20) that at high input SNR $\left(\mathrm{SNR}_{\text {in }} \gg\right.$ 1 ), the expression (20) can be approximated by $\mathrm{SNR}_{\text {out }}=$ $\mathrm{NSNR}_{\text {in }} / 4$. Conversely, at low SNR $\left(\mathrm{SNR}_{\text {in }} \ll 1\right)$, the expression (20) can be approximated by $\mathrm{SNR}_{\text {out }}=\mathrm{NSNR}_{\mathrm{in}}^{2} / 2$, the output SNR could be even worse than the input SNR. We can define the threshold of the algorithm as the interception point between the two limiting behaviors corresponding to the two cases of high and low SNR, obtaining the SNR threshold value equal to $\mathrm{SNR}_{\text {in }}=1 / 2$, about $-3 \mathrm{~dB}$. It is a constant and the length of signal does not influence the SNR.

Remark 5. Other transformations, such as the fractional Fourier transform and the Wigner-Hough transform, the threshold value is $1 / N$, this is advantageous because the threshold value can be lowered by increasing the number of samples [10]. While the threshold of the CPF applied to LFM signals is a constant, it is disadvantageous. The similar transformations include the polynomial-phase transform [11].

\subsection{Accuracy}

The performance of parameter estimation is usually evaluated by the statistical characteristics of the estimates. Here, the asymptotic statistical results are derived for all the estimated parameters based on the first-order perturbation analysis of maxima of random functions [12]. The following results can be obtained. 
Theorem 2. The mean-square error (MSE) of $\hat{\beta}$ is expressed by

$$
E\left\{(\delta \beta)^{2}\right\} \approx \frac{90}{N^{5}} \frac{1}{\mathrm{SNR}}\left(1+\frac{1}{2 \mathrm{SNR}}\right) .
$$

Theorem 3. The MSE of $\hat{\alpha}$ is expressed by

$$
E\left\{(\delta \alpha)^{2}\right\} \approx \frac{6}{N^{3} \mathrm{SNR}} .
$$

Theorem 4. The MSE of $\hat{b}$ is expressed by

$$
E\left\{(\delta b)^{2}\right\} \approx \frac{\sigma^{2}}{2 N} \text {. }
$$

Lemma 1 ([13]). For large $N$, the Cramer-Rao lower bounds of $\beta, \alpha$, and $b$ are expressed as

$$
\begin{aligned}
& \operatorname{CRLB}\{\hat{\beta}\} \approx \frac{90}{\mathrm{SNR} \cdot N^{5}}, \\
& \operatorname{CRLB}\{\hat{\alpha}\} \approx \frac{6}{\mathrm{SNR} \cdot N^{3}}, \\
& \operatorname{CRLB}\{\hat{b}\} \approx \frac{\sigma^{2}}{2 N} .
\end{aligned}
$$

If we normalize the variances by the corresponding CramerRao lower bounds, we obtain the efficiencies

$$
\varepsilon_{\beta} \approx 1+\frac{1}{2 \mathrm{SNR}}, \quad \varepsilon_{\alpha}=\varepsilon_{b} \approx 1
$$

Remark 6. The efficiencies of the Wigner-Hough transform (WHT) is [10]

$$
\varepsilon_{\beta}=\varepsilon_{\alpha} \approx 1+\frac{2}{\mathrm{NSNR}}+O\left(\frac{1}{N^{2}}\right), \quad \varepsilon_{b} \approx 1 .
$$

Compared with the CPF algorithm, the estimate accuracy of the chirp rate $\beta$ is higher than the CPF algorithm, and the estimate accuracy of the initial frequency $\alpha$ and the amplitude $b$ is the same as the CPF algorithm. But the implementation of the WHT requires 2D maximizations.

Remark 7. The efficiencies of the fractional Fourier transform (Frft) is [6]

$$
\varepsilon_{\beta}=\varepsilon_{\alpha} \approx 1+\frac{3}{2 N+1}+O\left(\frac{1}{N^{2}}\right), \quad \varepsilon_{b} \approx 1 .
$$

Compared with the CPF algorithm, the estimate accuracy of the chirp rate $\beta$ is higher than the CPF algorithm, and the estimate accuracy of the initial frequency $\alpha$ and the amplitude $b$ is the same as the CPF algorithm. But the implementation of the Frft still requires 2D maximizations.

\section{SIMULATIONS}

In this section, the Monte Carlo simulations are provided to support the theoretical results. In the experiment, the signal contains two components, the length of it is 255, and the parameters of each component are: $b_{1}=1, \alpha_{1}=\pi / 8, \beta_{1}=$ 0.005 , and $b_{2}=0.2, \alpha_{2}=\pi / 4$, and $\beta_{2}=0.002$. The sampling interval is 1 , the value of the input SNR varies from $-4 \mathrm{~dB}$ to $11 \mathrm{~dB}$ with an interval of $1 \mathrm{~dB}$, we run 200 Monte Carlo simulations, the measured MSEs $(\mathrm{dB})$ of each parameter and the theoretical MSEs (dB) just as (21)-(23) are shown in Figures 3 and 4.

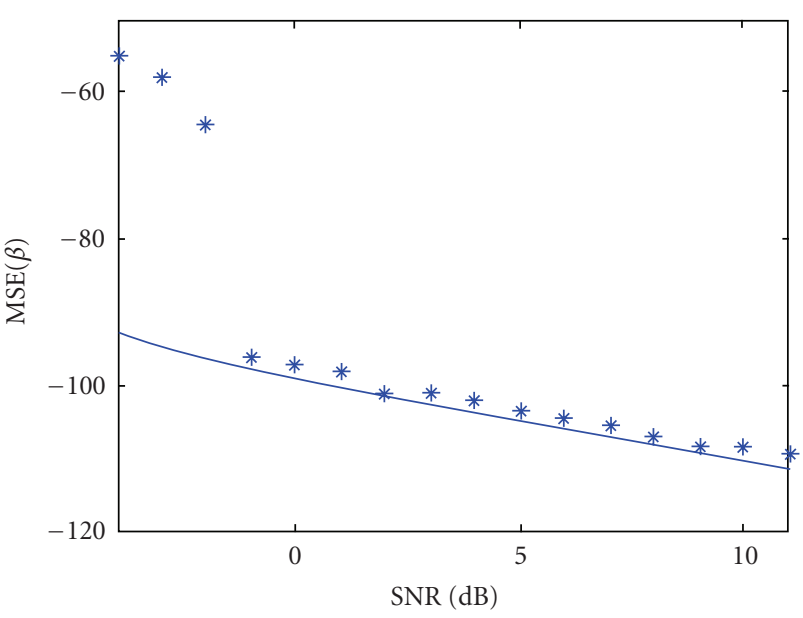

(a)

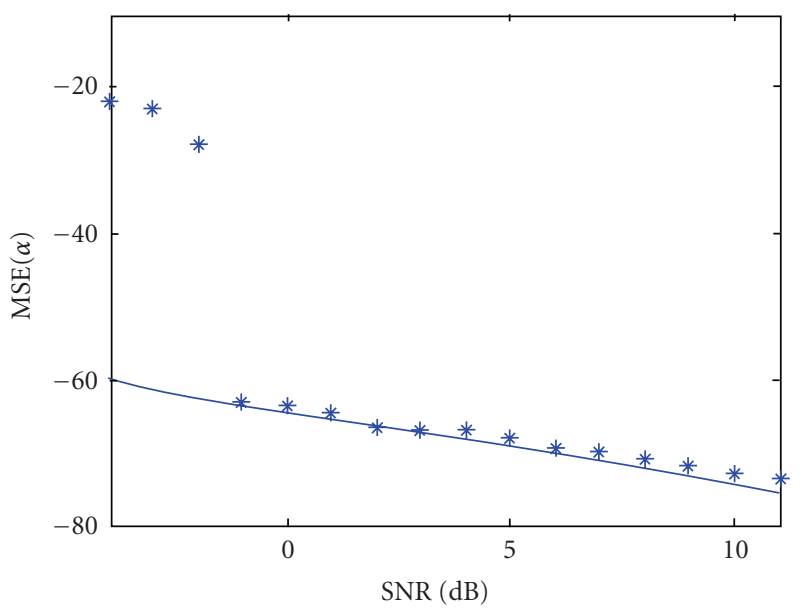

(b)

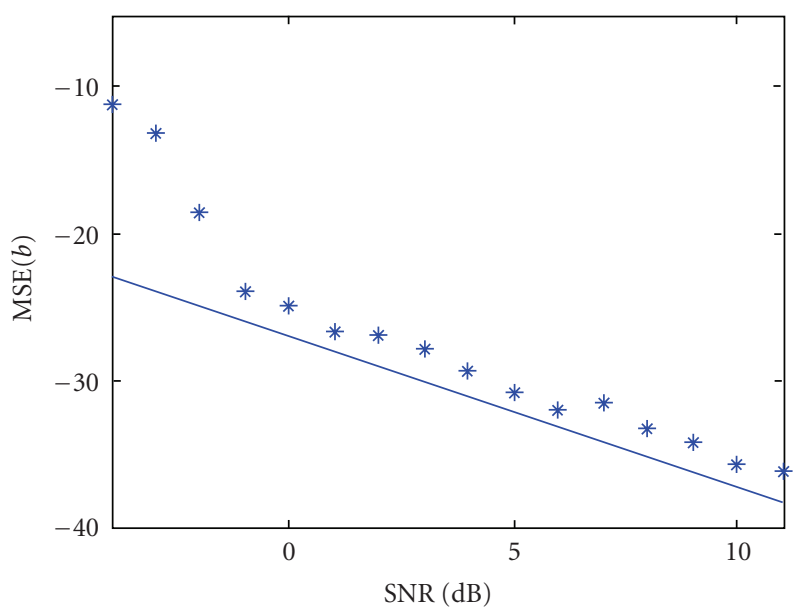

(c)

FIGURE 3: MSEs of parameter estimates of the first component. (Full lines are the theoretical MSEs, and circles indicate measured MSEs).

As shown in Figure 3, for the first component, when the SNR is higher than $-2 \mathrm{~dB}$, the measured MSEs and the theoretical MSEs are well matched. If the SNR is lower than $-2 \mathrm{~dB}$, there exist big errors between the measured MSEs 


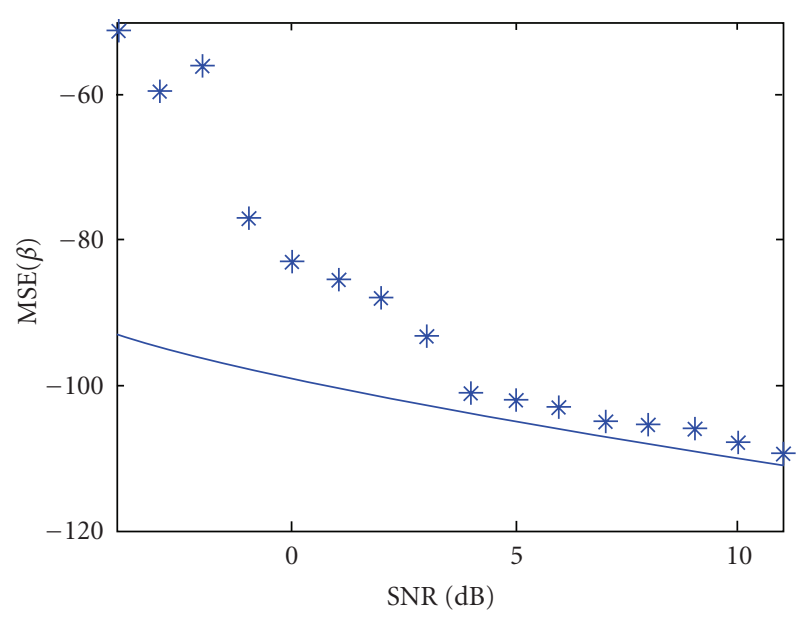

(a)

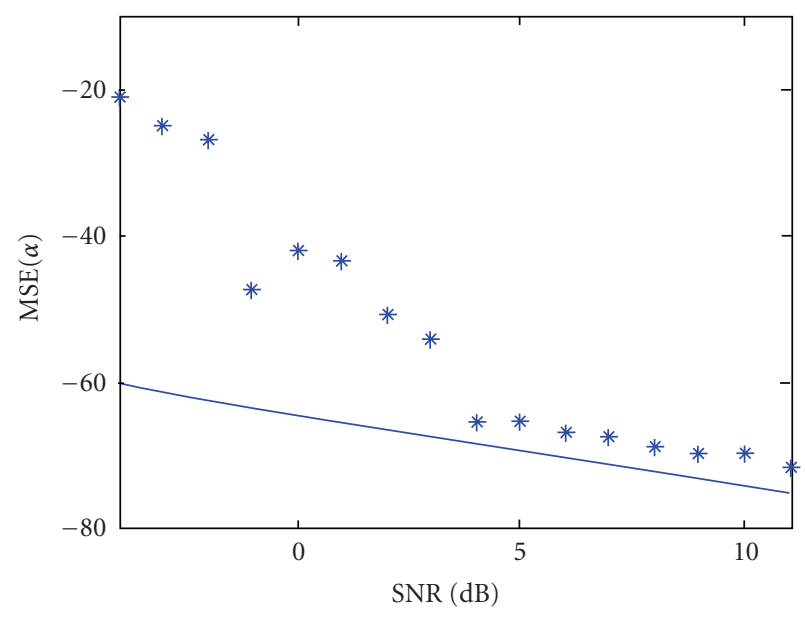

(b)

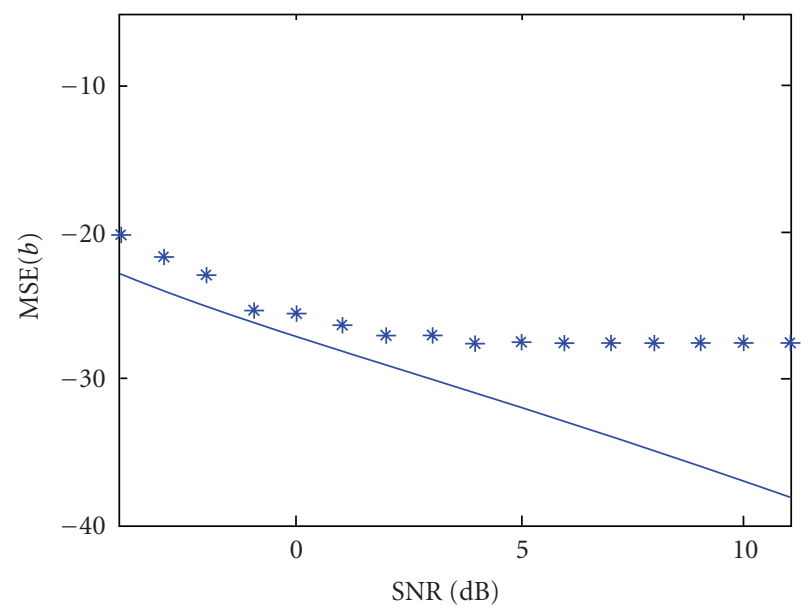

(c)

FIGURE 4: MSEs of parameter estimates of the second component. (Full lines are the theoretical MSEs, and circles indicate measured MSEs).

and the theoretical MSEs, this is because the approximations implied in the theoretical analyses are no longer valid at low SNRs. For the second component, we can see from Figure 4 that, after filtering out the first component by the CLEAN technique, the measured MSEs are close to the theoretical MSEs when the SNR is higher than $-2 \mathrm{~dB}$, but compared with Figure 3, the accuracy decreases in a way, and there are some irregular variations of the measured MSEs, this is because of the weakness of the second component compared with the noise at low SNRs, and the influence of the first component exists simultaneously.

\section{CONCLUSION}

The detection and parameters estimation of multicomponent LFM signal can be realized by the CPF. The principle of detection and parameters estimation of single LFM signal is presented. For multicomponent LFM signal, the CLEAN technique combined with the CPF is proposed to detect the weak components submerged by the stronger components. The statistical performance is analyzed in this paper and the simulation results demonstrate the validity of the algorithm proposed.

\section{REFERENCES}

[1] T. J. Abatzoglou, "Fast maximum likelihood joint estimation of frequency and frequency rate," IEEE Transactions on Aerospace and Electronic Systems, vol. 22, no. 6, pp. 708-715, 1986.

[2] R. M. Liang and K. S. Arun, "Parameter estimation for superimposed chirp signals," in Proceedings of the IEEE International Conference on Acoustics, Speech, and Signal Processing (ICASSP '92), vol. 5, pp. 273-276, San Francisco, Calif, USA, March 1992.

[3] I. Raveh and D. Mendlovic, "New properties of the Radon transform of the cross Wigner/ambiguity distribution function," IEEE Transactions on Signal Processing, vol. 47, no. 7, pp. 2077-2080, 1999.

[4] Y. Sun and P. Willett, "Hough transform for long chirp detection," IEEE Transactions on Aerospace and Electronic Systems, vol. 38, no. 2, pp. 553-569, 2002.

[5] M. Wang, A. K. Chan, and C. K. Chui, "Linear frequencymodulated signal detection using Radon-ambiguity transform," IEEE Transactions on Signal Processing, vol. 46, no. 3, pp. 571-586, 1998.

[6] L. Qi, R. Tao, S. Zhou, and Y. Wang, "Detection and parameter estimation of multicomponent LFM signal based on the fractional Fourier transform," Science in China Series F, vol. 47, no. 2, pp. 184-198, 2004.

[7] P. O'Shea, "A new technique for instantaneous frequency rate estimation," IEEE Signal Processing Letters, vol. 9, no. 8, pp. 251-252, 2002.

[8] P. O'Shea, "A fast algorithm for estimating the parameters of a quadratic FM signal," IEEE Transactions on Signal Processing, vol. 52, no. 2, pp. 385-393, 2004.

[9] J. Tsao and B. D. Steinberg, "Reduction of sidelobe and speckle artifacts in microwave imaging: the CLEAN technique," IEEE Transactions on Antennas and Propagation, vol. 36, no. 4, pp. 543-556, 1988.

[10] S. Barbarossa, "Analysis of multicomponent LFM signals by a combined Wigner-Hough transform," IEEE Transactions on Signal Processing, vol. 43, no. 6, pp. 1511-1515, 1995. 
[11] S. Peleg and B. Porat, "Estimation and classification of polynomial-phase signals," IEEE Transactions on Information Theory, vol. 37, no. 2, pp. 422-430, 1991.

[12] S. Peleg and B. Porat, "Linear FM signal parameter estimation from discrete-time observations," IEEE Transactions on Aerospace and Electronic Systems, vol. 27, no. 4, pp. 607-616, 1991.

[13] B. Ristic and B. Boashash, "Comments on "the CramerRao lower bounds for signals with constant amplitude and polynomial phase"', IEEE Transactions on Signal Processing, vol. 46, no. 6, pp. 1708-1709, 1998. 